\title{
Le respect : catégorie du social, catégorie du politique dans une favela de Recife
}

\author{
Dominique VIDAL
}

\section{(2) OpenEdition \\ 1 Journals}

\section{Édition électronique}

URL : http://journals.openedition.org/conflits/185

DOI : 10.4000/conflits. 185

ISSN : $1777-5345$

\section{Éditeur :}

CCLS - Centre d'études sur les conflits lilberté et sécurité, L'Harmattan

\section{Édition imprimée}

Date de publication : 1 septembre 1999

ISBN : 2-7384-8616-9

ISSN : 1157-996X

\section{Référence électronique}

Dominique VIDAL, «Le respect : catégorie du social, catégorie du politique dans une favela de Recife », Cultures \& Conflits [En ligne], 35 | automne 1999, mis en ligne le 16 mars 2006, consulté le 30 mars 2021. URL : http://journals.openedition.org/conflits/185; DOI : https://doi.org/10.4000/conflits. 185

Ce document a été généré automatiquement le 30 mars 2021.

Creative Commons License 


\title{
Le respect : catégorie du social, catégorie du politique dans une favela de Recife
}

\author{
Dominique VIDAL
}

1 À Brasília Teimosa, favela urbanisée de Recife au Nordeste du Brésil, la référence au respect (respeito) organise aussi bien l'évaluation des relations sociales que les jugements sur le personnel politique ${ }^{1}$. L'exigence de respect n'a certes rien de bien original. À en juger par sa fréquence dans les différends familiaux, les rapports de travail ou les conflits de voisinage, on pourrait même être tenté d'affirmer qu'il s'agit de l'une des choses du monde les mieux partagées ${ }^{2}$. Ne la retrouve-t-on pas chez des populations défavorisées de pays à la tradition démocratique nettement plus établie qu'au Brésil : les ouvriers anglais, les jeunes des cités françaises, les Noirs des ghettos nord-américains ou les dealers portoricains d'East $\mathrm{Harlem}^{3}$ par exemple ? La notion de respect revêt néanmoins un sens singulier à Brasília Teimosa. Elle manifeste d'abord le souci de ses habitants de voir reconnue leur pleine appartenance sociale, définie comme appartenance à l'humanité par opposition à la condition d'esclave. Elle révèle aussi l'intériorisation de valeurs et de normes des couches supérieures et l'identification à la place dévolue au peuple dans les constructions de l'identité nationale. En oscillant entre la préférence pour les formes anciennes de rapports hiérarchiques, qui assurent aux individus en situation d'infériorité la protection de dominants, et une demande d'égalité singulièrement ambivalente, elle traduit enfin l'indétermination de l'époque pour ces pauvres qui, par-delà la grande diversité de leurs trajectoires, partagent tout un ensemble de représentations collectives et vivent l'instauration de la démocratie dans un contexte de crise socio-économique particulièrement déroutant. En étudiant différents usages de la notion de respect dans cette favela de Recife ${ }^{4}$, on montrera que le fait politique ne peut jamais y être pensé sans référence constante à la spécificité du social tant le recours à des catégories morales de la quotidienneté imprègne le déchiffrement des activités politiques. Comprendre cette catégorie indigène, catégorie du social érigée en catégorie du politique, fait en effet apparaître tout un univers de significations occulté par les 
approches qui séparent l'étude des formes de vie de celle du rapport au politique ${ }^{5}$. Il faut ainsi connaître l'importance accordée à la qualité des relations sociales pour accéder au sens donné au statut de citoyen et prendre en compte la prégnance de moments politiques fondateurs pour interpréter certaines représentations sociales. Étroitement liés au contexte spatial et historique de la recherche, les résultats ici présentés ne se veulent toutefois nullement des énoncés de validité plus générale que seul le travail comparatif permet de formuler. Notre démarche entend plutôt montrer l'intérêt d'une approche ethnographique dans la compréhension sociologique du politique et de la construction démocratique. Voir son appartenance sociale reconnue Péninsule envahie en 1958 par des pauvres de Recife et des campagnes nordestines en quête d'un habitat meilleur, Brasília Teimosa abrite aujourd'hui autour de 17000 résidents et jouit, à Recife, d'une réputation de population particulièrement revendicative et bien organisée, qui tient au rôle attribué à ses associations d'habitants dans les mouvements revendicatifs ayant conduit à l'urbanisation du site ${ }^{6}$. Bien qu'une impression d'homogénéité s'en dégage de prime abord, la diversité des pratiques religieuses, l'hétérogénéité professionnelle et des différences de revenus notables entre groupes domestiques en font une population socialement diversifiée. À la différence de ces groupes fondés sur une communauté d'habitat dotée d'une forte identité collective et d'un dispositif normatif à grand pouvoir intégrateur auxquels nous a habitués la sociologie urbaine, ses habitants ne manifestent qu'un faible sentiment d'appartenance à un ensemble commun et n'établissent aucune frontière nette avec les couches supérieures dont ils partagent des pans entiers du système de valeurs. Le souci permanent de voir leur pleine appartenance sociale reconnue les rend incapables de construire leur identité tant personnelle que collective sans le regard des dominants. Centrale dans l'évaluation de la qualité des relations sociales, la notion de respect - et son corollaire, la dénonciation du manque de respect (falta de respeito) - leur permet de dénoncer ceux qui refusent de reconnaître leur pleine appartenance à la société. Car peu sont les résidents de la péninsule qui n'aient un jour éprouvé le sentiment d'être assignés dans une incommensurable différence lors d'interactions avec les membres des couches supérieures. Quelques mots pleins de sous-entendus, un geste de défiance ou un regard jugé fuyant suffisent souvent pour qu'ils se sentent profondément humiliés par la révélation de leur infériorité sociale. Ils se savent ainsi ravaler au rang infamant de délinquant ou de mendiant quand, à leur approche, de peur d'être volés ou de se voir demander une aumône, des passants agrippent leurs effets ou détournent subitement le regard. Le préjugé racial apparaît parfois de façon flagrante dans ces mises à distance. Ses manifestations les plus fréquentes - l'ordre d'emprunter l'ascenseur réservé aux domestiques dans les immeubles de standing et l'interdiction d'accès à certains établissements de loisirs - leur rappellent notamment le stigmate qui afflige le Noir dans une société où pèse encore le poids des catégories de classement de l'esclavagisme. Rares sont néanmoins ceux qui, à Brasília Teimosa, remettent en cause les inégalités abyssales de la société brésilienne et expriment une quelconque hostilité à l'encontre de ceux que l'on appelle les « riches ». Quand un jugement négatif est porté sur ces derniers, c'est bien moins l'inégalité économique qui est dénoncée que la façon dont elle est signifiée. Car ce n'est ni la richesse, ni un statut social supérieur que l'on reproche occasionnellement aux "riches", mais justement ces comportements dédaigneux, ce " manque de respect » si durement vécu. Néanmoins, pour virulents que soient parfois les propos sur les "riches ", on reconnaît aisément qu'il en existe aussi de «bons » qui «respectent les pauvres » et doivent par conséquent bénéficier en 
retour de leur pleine considération, de leur « respect». Le besoin de s'affilier à des individus au statut social plus élevé est au demeurant tel qu'il interdit l'apparition d'un antagonisme de classe à l'encontre des dominants. Trois types de liens permettent notamment de se penser comme appartenant pleinement à la société en dépit de la domination subie : l'adhésion aux modèles de la société de consommation, la relation à l'employeur et l'adhésion à une représentation du peuple originellement promue dans le cadre de la construction de l'identité nationale. Tous supposent la reconnaissance de l'appartenance sociale par le regard des dominants. Si manifeste et ouvertement revendiqué chez les jeunes, le désir de s'inscrire dans l'univers symbolique de la société globale transparaît dans l'influence des modèles culturels véhiculés par les moyens de communication de masse. Le développement de marques de vêtements bon marché permet ainsi à tous ceux qui le veulent de s'habiller selon la dernière tendance à défaut de véritablement masquer l'origine sociale. Le goût de la mode y étant du reste comme partout autant recherche que refus du conformisme, chacun cultive son " petit genre " qui le met à la page et le singularise. En se rendant sur la plage du quartier résidentiel de Boa Viagem plutôt que sur celles qui bordent la péninsule, bien des jeunes de Brasília Teimosa entendent exprimer leur similitude avec ceux des milieux favorisés, bien que peu de relations durables se nouent effectivement entre eux ${ }^{8}$. À la différence des Italo-Américains du West End bostonien qui, à la fin des années 1950, fuyaient tout contact avec les classes moyennes pour éviter les situations qui révélaient leur infériorité , les habitants de la péninsule recherchent au contraire leur fréquentation, fût-elle limitée ou symbolique. Ceux d'entre eux qui comptent de telles relations aiment à en faire largement état, quand bien même les liens tissés, s'ils se parent volontiers du vocabulaire de l'amitié, se révèlent généralement beaucoup plus fragiles qu'ils ne l'estiment. Avoir passé une soirée assis dans un bar à la même table qu'un individu jouissant d'un certain prestige social, que l'on a tutoyé et appelé par son prénom, ou l'avoir servi régulièrement dans une position subalterne, comme serveur dans un restaurant ou vendeur dans un magasin, suffit à beaucoup à se targuer de son amitié et à se livrer aux plaisirs de ce que les Anglo-saxons appellent, sarcastiquement, le name dropping. Une autre modalité du sentiment de pleine appartenance sociale se joue dans les relations entre employeur et employé. Si les rapports de travail peuvent être le lieu où la condition d'inférieur est la plus durement vécue, ils sont aussi celui où peut prévaloir un code de comportement paternaliste qui garantit certaines protections au subalterne. Ce code de comportement puise son origine dans les relations qui s'établissaient jadis entre maîtres et esclaves : en contrepartie de leur allégeance, les premiers donnaient aux seconds une identité sociale en les considérant comme des membres de leurs familles. Dans le Brésil esclavagiste, comme le note Katia Mattoso, "la survie même de l'homme noir dépend absolument de sa "repersonnalisation", d'une certaine acceptation de sa position dans la vie sociale. (...) L'insertion sociale de l'esclave, son acceptation par des hommes libres dans une société fondée sur le travail servile, va dépendre étroitement de la réponse que le travailleur-esclave donne à ses maitres sur le plan de la fidélité, de l'obéissance et de l'humilité » ${ }^{10}$. Il en résulte la forte ambivalence du sentiment du dominé envers le dominant, ambivalence aisément repérable dans les discours des employés sur leurs employeurs. S'ils condamnent catégoriquement toute forme de traitement qui rappelle les aspects dégradants du régime servile, ils n'en estiment pas moins qu'un patron a le devoir de protéger ses employés par la prise en charge de frais médicaux imprévus et par l'intervention auprès des autorités en cas de problèmes administratifs ou judiciaires. Aussi des 
relations qui, pour asymétriques qu'elles soient, n'en expriment pas moins une reconnaissance réciproque de chaque partie sont pleinement acceptées et valorisées. En restant toujours poli, en donnant des ordres ou en faisant des reproches sans élever la voix, le «bon patron» «traite bien» ses employés et leur montre qu'il les « respecte ». Et il est ainsi souvent bien davantage celui qui les considère comme des « amis » ou des membres à part entière de sa propre famille que celui qui les rémunère correctement. Pour affirmer leur pleine appartenance sociale, les habitants de Brasília Teimosa s'identifient également à un " peuple » source et garant de l'identité nationale. La force de l'attachement à la nation en milieu populaire n'a certes en elle-même rien de spécifiquement brésilien ${ }^{11}$. Ce qui est, en revanche, spécifique au Brésil, c'est le fait que la mobilisation des secteurs populaires autour de l'idée nationale ne s'est pas opérée par leur participation au processus politique, mais à partir d'une représentation du «peuple» proposée par des élites politiques et intellectuelles dans un contexte marqué par le refus du libéralisme politique ${ }^{12}$. Dans les années 1925-1940, période de rejet des modèles étrangers, toute une génération d'intellectuels et d'artistes découvrent le fondement de ce que l'on nomme alors la "réalité brésilienne » dans les pratiques et les manières d'être des milieux populaires dont l'hétérogénéité avait jusque là était perçue comme un obstacle à la construction de la nation. Sont reconnus comme éléments fondateurs de la société et de la culture brésiliennes les Indiens, les populations pauvres des zones rurales puis, enfin, les Noirs avec la publication, en 1933, de Maîtres et esclaves de Gilberto Freyre. Le métissage n'est plus vu comme une tare, mais comme une richesse, et naît à ce moment-charnière de l'histoire brésilienne ce que d'aucuns appelleront plus tard le «mythe des trois races " qui fait du Brésilien un homme nouveau, syncrétique, produit par le mélange du Portugais, de l'Indien et de l'Africain. Qu'il s'agisse dans ces constructions du type de relations qui existaient sur les plantations ou du tempérament «cordial» du Brésilien, l'affectivité dans les rapports interpersonnels est tenue comme fondement du lien social et élevée en trait spécifique de l'identité nationale. Il n'est ainsi personne à Brasília Teimosa qui n'ait intériorisé ces représentations partagées du reste à tous les degrés de la structure sociale et n'affirme résolument son appartenance au "peuple", socle de l'identité brésilienne. Et bien que l'appartenance sociale puisse être remise en cause dans la moindre interaction avec les couches supérieures, nul n'en demeure pas moins toujours convaincu d'être un "Brésilien » à part entière. Le carnaval est un de ces moments d'affirmation de la place fondatrice du peuple dans l'identité brésilienne. À Brasília Teimosa, on considère ainsi que sans sa participation le carnaval perdrait tout son sens, seuls les secteurs populaires pouvant donner une interprétation authentique des musiques et des danses carnavalesques. Pour stéréotypées qu'elles soient, ces représentations de l'apport du peuple à la culture brésilienne ne sont pas dénuées de fondements. Qui connait l'histoire des styles musicaux et de danse brésiliens sait en effet que ceux-ci résultent largement de l'incorporation d'éléments empruntés aux pratiques culturelles populaires. Fête à l'origine dérivée de l'Entrudo portugais, réservée aux élites et interdite aux Noirs, le carnaval connût une profonde transformation dans les années 1930 avec son ouverture au peuple à la faveur de la reconnaissance de son apport fondamental à la culture nationale ${ }^{13}$. Sa contribution décisive à la fête carnavalesque devient à ce point évidente qu'il semble aujourd'hui à tous qu'il en est à l'origine même. Aussi, par leur participation au carnaval, les couches populaires ne font en réalité que jouer le rôle qui leur a été dévolu dans les constructions de l'identité nationale. On voit une fois encore à ce propos combien leur 
appartenance sociale étant reconnue au travers de leur apport à cet événement symbole de l'identité brésilienne, de ce spectacle donné aux autres et avec les autres, la construction de l'identité collective des plus démunis dépend du regard des secteurs sociaux favorisés. Dire que «le Brésil est le pays du carnaval» et que "sans la participation du peuple, il n'y a plus de carnaval », c'est affirmer que « le peuple » est bien la source de l'identité nationale, le sujet de la nation. Et de cette certitude d'être une composante fondamentale de l'identité nationale, il en va également de la profonde conviction de nombreuses jeunes femmes d'incarner parfaitement - et surtout mieux que les femmes des couches supérieures - «la femme brésilienne » (mulher brasileira) à la sensualité célébrée dans cette célébration annuelle de la brésiliennité qu'est le carnaval, quitte à accepter ce rôle de "petit animal joli et amusant " élément de l'«ambiguïté des rapports interethniques» au Brésil ${ }^{14}$. La fragilité des solidarités locales L'intériorisation de certaines parties du système de normes et de valeurs des dominants pèse aussi sur les rapports sociaux à Brasília Teimosa. La dénonciation constante du manque de respect entre ses habitants constitue un indice de la fragilité du lien social sur une péninsule où, vivant dans un monde instable, chacun ne doit bien souvent compter que sur lui-même pour trouver une solution à un problème. À s'en tenir à leurs propos sur les obligations morales devant régir les supposés collectifs formés par les parents, les voisins et les amis, on pourrait pourtant se laisser convaincre que ses habitants forment une Gemeinschaft au sens donné à cette catégorie par Ferdinand Tönnies, c'est-à-dire une "communauté de sang, de lien, d'esprit » reposant sur " la parenté, le voisinage, l'amitié » ${ }^{15}$. Quand ils disent ainsi qu'il revient à chacun de "remplir ses devoirs » (cumprir seus deveres), qu'il «en va de l'obligation [de quelqu'un] " (é a obrigação) de faire telle ou telle chose, qu' "il peut » (pode) ou qu' « il ne peut pas » (não pode) faire ceci ou cela, ou qu'untel « a tort » (está errado) ou "a raison" (está certo), ils en appellent à des règles s'imposant à chacun. Tels qu'ils sont énoncés, ces différents codes de comportement paraissent se compléter et pouvoir gouverner l'ensemble des relations possibles. Le lien social semble procéder de la subordination des individus aux règles nécessaires au fonctionnement pérenne d'une totalité organique leur préexistant, et non pas d'un contrat fondant le collectif. Lors de l'évocation de ces règles, le système juridique n'est en effet mentionné que s'il recoupe ces obligations définies à même le social et paraissant issues d'une loi naturelle. L'étude de la quotidienneté révèle néanmoins un écart important entre ce discours normatif et son observation dans les faits ${ }^{16}$. C'est sans nul doute dans les rapports entre parents que le contraste entre les obligations censées gouverner les relations entre membres d'un même collectif et la précarité de leur observation apparaît dans toute son ampleur. La solidarité est des plus variables dans la parentèle, où la solidité des liens du sang contraste notamment avec la précarité des liens conjugaux ${ }^{17}$. Il est peu de foyers qui ne comptent une femme élevant seule un enfant, beaucoup de géniteurs préférant abandonner leurs compagnes plutôt que de subir l'indignité qui frappe celui qui, par son travail, n'assume pas les responsabilités incombant au chef de famille. Quand les ressources le permettent, on secourt généralement les parents résidant sous le même toit ou dans le quartier. Mais quand ceux-ci vivent dans d'autres villes, l'aide s'avère plus aléatoire, bien que des familles dans le besoin reçoivent parfois des mandats postaux de parents établis à des milliers de kilomètres. Il suffit du reste bien souvent qu'un conflit passé (une accusation de tentative de séduction du conjoint ou d'un enfant, un héritage mal réglé ou la faillite d'une petite affaire commerciale dans la plupart des cas) ait mis un terme aux liens 
familiaux pour qu'un individu indigent ne puisse espérer un quelconque soutien. Les relations de voisinage diffèrent aussi du code de comportement énoncé à leur sujet. Dès la première visite au quartier, on ne peut qu'être frappé par les précautions prises par chaque foyer pour se protéger : quand l'accès au domicile n'est pas défendu par de hauts murs au-dessus desquels des pointes en fer ou des tessons de bouteilles ont été coulés dans le ciment, des grilles protègent presque toujours les fenêtres. L'extrême proximité des habitations rend en réalité les relations de voisinage toujours grosses de différends. Qu'un bien prêté soit perdu ou détérioré, qu'un arrangement ne se réalise pas dans les termes initialement prévus ou que des propos sur la moralité d'une femme aient été tenus dans une discussion d'après-boire suffisent à ce que des voisins en viennent à ne plus se parler. En outre, plus encore que dans le cas des parents, l'aide des voisins en cas de difficulté varie selon la qualité des liens entretenus. Si des aliments ou des fonds peuvent être régulièrement collectés pour une famille du voisinage jusqu'à ce qu'elle connaisse meilleure fortune, une autre, tenue en piètre estime, peut très bien ne rien recevoir. Les amis n'entretiennent pas de même des relations aussi solides et exclusives que celles que leur prête la rhétorique de l'amitié. Si beaucoup d'hommes fréquentent assidûment les buvettes de coins de rues, la sociabilité masculine ne prend jamais la forme d'une institution telle que le cuatismo mexicain, groupes d'hommes (los cuates) engagés dans des relations amicales fondées sur des obligations mutuelles très contraignantes et la pratique régulière de beuveries collectives $^{18}$. Comme dans le cas des parents et des voisins, la solidarité attendue des amis rencontre vite ses limites, leur situation financière les empêchant souvent d'accorder l'aide demandée. Face aux aléas de l'existence, la faiblesse et la précarité de leurs ressources placent donc la plupart des habitants de Brasília Teimosa dans une situation de grande vulnérabilité. Quand un problème ne peut être résolu par des parents, des voisins ou des amis, beaucoup parviennent à mobiliser des soutiens dans d'autres réseaux personnels. Contrairement aux pauvres mexicains et portoricains étudiés par Oscar Lewis, peu d'entre eux sont isolés au point de n'avoir pas de rapports avec le reste de la société ${ }^{19}$. Bien au contraire, nombreux sont ceux qui, même si l'essentiel de leurs relations sont centrées sur le quartier, savent tirer parti de liens tissés avec des individus n'y résidant pas pour trouver une aide ponctuelle. Face à une difficulté, chacun se retrouve en réalité dans une situation singulière définie par la spécificité de son insertion sociale. Il lui faut alors passer en revue ceux dont on peut espérer un secours : un parent, un voisin, un ami, un collègue de travail, un fidèle de la même église, l'employeur, un ancien employeur, l'employeur d'un ami, d'un parent ou d'un voisin, ou d'un de leurs amis, de personnes connues dans les événements les plus divers. À la différence des classes populaires anglaises décrites par Hoggart où la conscience d'un antagonisme radical entre " eux » et " nous » conduisait à ne solliciter l'aide des couches supérieures qu'en ultime recours, la valeur accordée aux formes d'affiliation aux dominants inscrit au contraire de telles demandes dans le cadre des relations devant prévaloir entre "riches » et "pauvres ${ }^{20}$. Connaître un professeur dans un établissement privé, une infirmière dans un service hospitalier ou un avocat peut permettre de bénéficier d'un abattement sur les frais de scolarité, d'obtenir rapidement une consultation ou de bénéficier d'un conseil opportun pour percevoir une indemnisation pour licenciement abusif. Le préjugé racial S'il affecte peu les relations ordinaires, le préjugé racial à l'encontre des Noirs se manifeste néanmoins régulièrement quand survient un litige sur le manque de respect. Il traduit une autre forme de l'intériorisation de critères de classement des dominants et du refus de ce qui 
rapproche de la condition d'esclave. Au moindre différend, quand quelqu'un, se classant Blanc ou métis, estime qu'un individu qu'il considère Noir lui " a manqué de respect ", une invective à l'égard des Noirs lui permet d'affirmer son bon droit en s'en démarquant socialement par l'invocation d'une différence de nature radicale. Celui ou celle avec qui l'on entretenait jusqu'alors des rapports cordiaux et que l'on considérait comme un ami devient soudainement un "sale Nègre" ou une "sale Négresse". Prenant l'entourage à témoin, celui qui s'estime lésé ou juge qu'on lui a manqué d'égards soutient qu' "avec les Noirs, c'est toujours pareil, ils ne valent rien ». Pour donner force à l'argumentation, des éléments du stigmate frappant les Noirs sont fréquemment rappelés. Si la personne objet du ressentiment est un homme, sa motivation au travail et son honnêteté seront mises en doute. ( « Un Nègre ne sait rien faire, même les ânes sont plus intelligents qu'eux »; «Tous sont analphabètes "; « Tous volent, y'en a juste certains qui volent moins que les autres »). Si c'est une femme, son comportement sera expliqué par une perversité sexuelle d'origine diabolique ("Elles ne pensent qu'à ça »; "Même la Négresse mariée cherche à tromper son mari »; "Elles rendent l'homme fou seulement pour lui prendre son argent»). Le préjugé racial se révèle particulièrement dans le cas des conflits de voisinage. Les familles noires sont alors accusées de se livrer à des actes de sorcellerie et de jeter le mauvais sort sur leurs ennemis. De même, quand une jeune mère élève seule dans la plus grande difficulté un enfant à la peau plus foncée qu'elle, il se trouve souvent une mauvaise langue pour dire que «c'est bien triste, c'est sûr, mais qu'elle n'avait qu'à pas coucher avec un Noir ". Ces jeunes femmes nient généralement la couleur du père parti en affirmant mordicus qu'ils étaient aussi blancs qu'elles et que le teint de leurs enfants relève des mystères de la génétique. Le stigmate constitué par une peau noire est d'ailleurs tel que, lors d'une visite à un nouveau-né, la couleur du bébé et son évolution possible font généralement l'objet des premiers commentaires. Réunis autour du berceau, au vu de son apparence, parents, amis et voisins supputent sur sa teinte définitive qui ne sera définitivement acquise que passées plusieurs années. Dans une population largement métissée, on ne pourrait qu'être surpris de l'existence de ce préjugé si le système de classification raciale en vigueur ne présentait la particularité de permettre à un individu apparemment noir de pouvoir prétendre se différencier radicalement des Noirs. Pour primordiale qu'elle soit dans l'attribution de l'identité raciale, la couleur de la peau n'est en effet pas le seul élément définissant un individu comme Noir. La texture du cheveu est pratiquement aussi importante que la couleur de la peau: selon que ces cheveux sont lisses ou crépus, un enfant apprend très jeune qu'il a les " cheveux bons " (cabelo bom) ou les «cheveux mauvais» (cabelo ruim). Des critères de classification sont fréquemment invoqués à Brasília Teimosa pour refuser catégoriquement d'être considéré Noir ${ }^{21}$. De même, les quelques jeunes, membres de groupes folkloriques et amateurs de reggae, revendiquent un héritage africain et une identité noire ne rencontrent guère d'audience, à la différence des vigoureuses organisations noires de Salvador da Bahia qui sont parvenues à susciter un sentiment de négritude parmi la population d'origine africaine ${ }^{22}$. L'interprétation de l'urbanisation L'intériorisation de normes des dominants apparaît aussi dans l'insistance à souligner le changement de statut urbain de Brasília Teimosa depuis son urbanisation. Y voyant une preuve de l'évolution sociale de sa population, ses habitants l'invoquent notamment pour se différencier des résidents des favelas (les favelados). Ils appliquent ce faisant les formes de mise à distance qu'ils subissent et se distinguent radicalement d'individus qui, à s'en tenir à des critères économiques, sont leurs semblables. Dans ces représentations, le 
parallèle entre le Noir et le favelado maintenu aux limites de la société revient souvent dans la phrase à valeur de maxime Favela é lugar de negro ("Une favela, c'est un endroit de Noirs »), qui rappelle la présence massive d'anciens esclaves dans les premières favelas des mornes de Rio de Janeiro. La construction de maisons en briques à la place de baraques de planches, l'ouverture de plusieurs écoles publiques et l'asphaltage des rues ont, selon eux, fait perdre le statut de favela à Brasília Teimosa pour en faire un quartier, appartenant de plein droit à la ville ${ }^{23}$. La présence d'associations d'habitants est aussi tenue comme un critère différenciant Brasília Teimosa d'une favela, en dépit pourtant de la faiblesse de la participation associative essentiellement motivée par des raisons instrumentales au moment de l'enquête. Ces organisations semblent témoigner de la vie en bonne intelligence de ses résidents, capables de s'unir pour revendiquer, à la différence de ceux des favelas que l'on présente comme des lieux anomiques où aucune action concertée ne peut voir le jour. Surtout, bien que très peu d'habitants de Brasília Teimosa aient activement participé au mouvement revendicatif à l'origine de son urbanisation, une grande majorité se prévaut de la réputation de population mobilisée et bien organisée dont les médias se font régulièrement l'écho ${ }^{24}$. La scolarisation de la quasi-totalité des enfants de la péninsule est d'ailleurs perçue comme un signe indubitable de l'entrée de sa population dans la société moderne, à la différence des favelados dont les manières trahiraient en permanence la grossièreté des paysans incultes. On observe là encore une autre différence de taille avec la classe ouvrière anglaise à laquelle, ces vingt dernières années, les pauvres urbains brésiliens ont été fréquemment comparés. Contrairement à ces quartiers ouvriers du nord de l'Angleterre où le goût de la lecture passait pour un «truc de bonnes femmes » et où celui qui a fait des études était amené à se couper de son milieu d'origine ${ }^{25}$, le détenteur de titres scolaires jouit à Brasília Teimosa de la considération de son entourage, quand bien même si sa situation financière est loin de l'en distinguer. Dans une société où l'accès à l'école des Noirs et des affranchis était interdit sous le régime servile et où le travail manuel, longtemps réservé aux esclaves et aux plus démunis, a toujours été méprisé, la possession de diplômes est un des critères les plus évidents de différenciation sociale, comme en témoigne l'exclusion des analphabètes du droit de vote jusqu'en 1985. L'on ne relève ainsi chez les jeunes de la péninsule aucune forme de cette "culture anti-école» (counter-school culture) qui traduit, en Angleterre, un des aspects de la résistance symbolique aux dominants de la culture ouvrière anglaise chez les enfants d'ouvriers et provoque à la fois l'acceptation des rôles subalternes et la régénération de cette classe ${ }^{26}$. Au contraire, avec cet espoir de la gratification différée si fort dans ce milieu populaire, ceux qui poursuivent, généralement au prix de gros sacrifices familiaux et personnels, des cursus scolaires et universitaires n'hésitent pas à s'affirmer supérieurs à ceux « qui ne veulent rien dans la vie ». S'il y a au demeurant une valeur largement partagée à Brasília Teimosa, c'est bien l'aspiration à l'ascension sociale, et ses habitants qui y parviennent sont généralement l'objet de commentaires élogieux pourvu qu'ils témoignent de leur « respect » des plus démunis en ne se montrant pas condescendants et oublieux de leurs « obligations » de «riches» quand une demande d'aide leur est adressée. Le rejet de la condition de favelado au nom de l'affirmation du statut de quartier de Brasília Teimosa conduit enfin ses habitants à stigmatiser certaines parties de la péninsule qui jurent avec l'image qu'ils entendent présenter d'eux-mêmes. C'est notamment le cas des résidents d'habitations sur pilotis le long du rivage, des constructions qui ne rappellent que trop la physionomie du lieu avant son urbanisation. Y compris pour ceux qui y ont des 
parents, ils forment un groupe à part désigné sous les noms de "gens du bord de mer " ou de "peuple de la plage ", et bien rares sont ceux qui n'ont une histoire de viol, de meurtre ou de trafic de stupéfiants à raconter à leur sujet, même si ces allégations s'étayent sur peu de faits avérés. Mais on leur reproche surtout en réalité de ne pas avoir accompagné l'évolution sociale de la péninsule en revenant vivre sur le rivage après avoir revendu des logements attribués gratuitement dans le cadre des programmes d'urbanisation, bien que, là encore, tout indique que les cas de ce genre soient infiniment moins nombreux que ne le veut la rumeur. Les différents usages du respect: un signe de l'ambivalence de l'époque Aucune culture communautaire ne compense à Brasília Teimosa la domination subie par la valorisation de l'appartenance à un groupe doté d'une image positive permettant l'affirmation d'une identité personnelle ou collective. Au contraire, l'incapacité de ses habitants à être assurés de leur pleine appartenance sociale sans le regard des dominants menace sans cesse les identités personnelles et rend particulièrement vulnérable leur identité collective. Parce qu'ils ont intériorisé les normes des couches supérieures, ils établissent entre eux des classements reprenant les principes de hiérarchisation dont ils pâtissent, et jamais ne peuvent se définir de façon autonome comme groupe ou membres d'une catégorie sociale spécifique. S'ils affirment vigoureusement appartenir au " peuple », ils disent davantage leur expérience au travers de schèmes interprétatifs de la société globale. Et ce qui les différencie des autres secteurs sociaux, ce sont en définitive bien moins des différences culturelles que de faibles ressources qui limitent leur participation sociale et les empêchent d'adopter un autre mode de vie. Peut-on alors caractériser sociologiquement la population étudiée ? La réponse n'est pas aisée tant sa diversité est grande ; elle est même impossible si on entend en proposer une image unifiée et stable. Les différents usages de la notion de respect invitent plutôt à souligner l'ambivalence qui la domine au moment historique de l'enquête. C'est-à-dire à relever combien, dans un univers désorganisé, la précarité des situations favorise la persistance d'une représentation holiste de l'ordre social aux dépens de la pleine acceptation de l'idéal d'égalité de la démocratie moderne. La revendication de respect exprime en effet autant une préférence pour les formes anciennes de rapports hiérarchiques entre dominants et dominés qu'une demande d'égalité elle-même complexe. Si leur caractère mouvant selon les situations rend particulièrement difficile la spécification des relations de la notion de respect avec le principe hiérarchique et l'idée d'égalité, on peut néanmoins analytiquement repérer trois configurations dans la subjectivité des acteurs. La première renvoie à une signification du respect qui exprime la recherche de rapports hiérarchiques dans le cadre d'une représentation organique de la société. L'idée d'égalité n'y a pas sa place. Pour les individus en situation d'infériorité, le sentiment de pleine appartenance sociale dépend alors de la protection de dominants. Le comportement paternaliste de l'employeur envers ses employés est la meilleure illustration de cette attente. Type de relation hérité du monde rural, l'allégeance au patron en contrepartie d'une place assignée permet au subalterne de construire son identité personnelle dans un univers où la menace du rejet aux marges de la société ne le quitte jamais. Quand rien n'est venu substituer cet ancien mode d'inscription dans l'ordre social pourvoyeur d'identité, quand la conjoncture ne laisse guère entrevoir une amélioration des conditions de vie, la relation paternaliste, forme de domination et rapport de dépendance personnelle, n'en est pas moins préférée à l'absence de liens avec les dominants ${ }^{27}$. La seconde configuration repose sur l'affirmation de la commune humanité de tous les membres du corps social, affirmation qui procède, on l'a vu, du 
rejet de tout ce qui évoque la condition d'esclave. Elle présuppose la reconnaissance d'une certaine forme d'égalité entre les individus sans pour autant que soit abandonnée une vision hiérarchique du social. La demande d'égalité qui s'y exprime s'apparente à l'idée d'«égalité civile» telle qu'elle a été historiquement formulée dans le prolongement du principe chrétien d'égale dignité des hommes devant Dieu. Synonyme ici de la notion de respect, le thème chrétien de la dignité de la personne humaine organise d'ailleurs bien des dénonciations du sort injuste fait aux plus démunis. Avec l'instauration d'un régime démocratique, l'ambivalence de l'appel au respect atteint peut-être ici son point le plus élevé. Cette conception de l'égalité peut en effet tout aussi bien sous-tendre l'affirmation du primat de l'individu sur le tout social, au fondement de la vision moderne de la démocratie, que traduire la vigueur d'une représentation organique et hiérarchique de la société. Dans une troisième configuration, l'exigence de respect traduit le refus d'une société hiérarchique où les places sont originairement assignées. Plus que de commune humanité des dominants et des dominés, c'est de "similitude des hommes" au sens tocquevillien qu'il est question ${ }^{28}$. Le respect ne se déploie plus ici à partir de positions précises, mais suppose au contraire que soit reconnue l'identité absolue des individus, ce que l'auteur de la Démocratie en Amérique nomme l'égalité des conditions, ce trait structurel des sociétés démocratiques où le travail permanent de réduction de l'altérité entre les hommes doit progressivement effacer les distinctions de nature héritées des constitutions aristocratiques. Refus en acte d'une société où chacun reçoit sa condition à sa naissance, les espoirs d'ascension sociale témoignent de ce désir d'une société de mobilité où aucune différence d'essence ou d'appartenance n'interdit d'accéder à une position convoitée. Certaines identifications aux modèles culturels de la société globale attestent pareillement de la recherche d'une uniformité symbolique marquant la ressemblance de tous par-delà les inégalités économiques. Quand les individus sont ainsi pensés comme fondamentalement mobiles, détachés de tous liens préalables non choisis et radicalement égaux en nature, c'est une demande d'égalité au sens moderne du terme qui s'énonce : cette forme d'égalité qui est au principe de l'idée de citoyenneté politique de la démocratie. Aucune de ces configurations ne se stabilise jamais au point d'effacer les autres. Leur co-présence fait l'originalité de la notion de respect dans une population confrontée au changement social et à la transformation politique dans une période d'incertitude. Au cœur de relations fondées aussi bien sur un principe hiérarchique («riche »-« pauvre», employeur-employé, mari-épouse, parent-enfant) que sur l'égalité (entre collatéraux, amis ou voisins), la référence au respect, principe de gestion de l'inégalité ou affirmation de formes diverses de l'égalité entre les individus, témoigne simultanément du poids de l'histoire, manifeste dans la vigueur des formes de hiérarchisation sociale à l'œuvre sous l'esclavagisme, et de la nouveauté du moment présent, révélée dans la reconnaissance de l'individu indépendamment de ses appartenances préalables ${ }^{29}$. Centrale dans l'évaluation de maintes interactions, la notion de respect exprime ce qui fait lien social pour ces individus en situation d'infériorité. Elle leur permet de dénoncer ceux qui, par une attitude ou une parole, semblent refuser de reconnaître leur pleine appartenance à la société ou de distinguer ceux qui ont une place dans l'ordre social, fût-ce en position subalterne, de ceux qui s'en trouvent rejetés du fait d'un comportement violant les règles censées assurer la préservation du lien social. Si peu suffit à ces pauvres de Recife pour qu'ils s'estiment refoulés à la frontière de la société, peu suffit aussi pour qu'ils se sentent "respectés ». Et c'est ainsi que, à tout moment, le lien social révélé et garanti par le respect peut être 
menacé de dissolution ou, inversement, susceptible de se (re)former. Le respect, socle du statut de citoyen Le terrain réserve parfois d'étonnantes surprises. Parti étudier le sens donné à la citoyenneté par une population longtemps tenue en retrait du processus politique, j'en eus une de taille quand je découvris que, à Brasília Teimosa, le mot « citoyen» (cidadão) appartenait au langage courant. On se saluait par des « Oh, citoyen »; on parlait du "citoyen qui a tout perdu», du «citoyen maltraité par la police "; on remettait un objet à un voisin sur les simples mots de " pour le citoyen " . Mais chose plus surprenante encore, ce mot était aussi de ceux dont le sens ne faisait aucun doute, une autre véritable catégorie indigène au contenu bien plus précis que le polysémique «respect». Quand, invoquant ma condition d'étranger pour affirmer l'ignorer, je demandais à quelqu'un de le définir, après qu'il l'eut ou non préalablement prononcé, on me répondait toujours sur le mode de l'évidence en des termes similaires. Le cidadão est " une personne de bien », qui n'a jamais eu "affaire à la police », qui entretient de «bonnes relations » avec autrui, quelle que soit sa position sociale, et qui " remplit ses obligations ", en pourvoyant aux besoins des siens par son travail si c'est un homme, en veillant à la bonne marche du foyer si c'est une femme. Soit une représentation du statut de citoyen au cœur de laquelle on retrouve sans peine justement les principaux éléments de la notion de respect et son ambivalence fondamentale. Car n'est un citoyen que celui dont le comportement atteste de son respect d'autrui. Et, de la même façon que le respect ne s'obtient qu'au travers d'autrui, n'est citoyen que celui qui est reconnu comme tel par les autres « citoyens ». Fait du reste remarquable, jamais les définitions recueillies du mot cidadão ne mentionnaient la dimension proprement politique de la citoyenneté (un mot qui n'est, par contre, jamais prononcé et ne fait pas sens), et ce alors que le thème de l'accès à la citoyenneté des plus démunis par leur participation aux affaires publiques organise les discours politiques depuis l'amorce de la transition démocratique. L'analyse des «droits » et des "devoirs» du citoyen dont il est souvent question à Brasília Teimosa permet de préciser la spécificité du rapport au politique de sa population et de l'importance accordée à la reconnaissance de la pleine appartenance sociale des individus en situation d'infériorités ${ }^{30}$. Le "droit de " (direito de) désigne essentiellement les droits civils et politiques. De très loin les plus mentionnés, les « droits » (direitos) définissent, eux, pour l'essentiel l'ensemble des prestations garanties par le droit du travail. Réalisée plus d'une décennie après la recherche de Caldeira dans une toute autre région du Brésil, notre enquête permet de prolonger et d'affiner son analyse en relevant chez les résidents de la péninsule la référence à différents moments d'accès aux droits sociaux des secteurs populaires. L'accès d'une partie des secteurs populaires urbains à des formes de protection sociale (mise en place d'un droit du travail, de systèmes de retraite et d'assurance-santé) sous les présidences de Getúlio Vargas constitue chez les plus de soixante ans un événement qui met véritablement fin à l'esclavage, qui avait pourtant été officiellement aboli près de cinquante ans auparavant ${ }^{31}$. À une époque où les formes de domination qui rappellent l'esclavage paraissent devoir être à jamais dépassées, ce lien qui se tisse dans les années 1930 entre le pouvoir et le peuple a valeur de moment fondateur dans la mémoire collective des secteurs populaires brésiliens qui y voient la reconnaissance de leur pleine appartenance sociale. À telle enseigne que les travailleurs agricoles qui n'ont accédé au droit du travail que bien après la mort de Vargas en parlent dans des termes identiques aux citadins qui en ont bénéficié de son vivant, et que bien des jeunes, sous une forme atténuée et sans jamais distinguer le dictateur du président démocratiquement élu, louent aujourd'hui encore ses mérites 
tant vantés par leurs ascendants ${ }^{32}$. Un autre type de «droits du citoyen » est également évoqué. Ils renvoient aussi bien aux conditions de logement (le droit à l'électricité, le droit à l'égout), à l'existence de services publics (le droit à l'éducation, le droit à la santé) qu'à des exigences plus générales comme le droit au travail ou le droit à la sécurité. On repère ici les traces du langage des organisations de base qui, lors de transition à la démocratie, faisaient de la référence aux «droits » un mode majeur de formulation des demandes aux acteurs gouvernementaux ${ }^{33}$. La notion de respect fait alors souvent l'objet d'une mise en forme juridique, lorsque les diverses demandes excipent d'un droit plus fondamental encore : le «droit au respect» ou le «droit à la dignité ». Fréquemment formulé par des acteurs issus du catholicisme progressiste, ce droit-là renvoie explicitement à l'idée chrétienne d'une égale dignité des hommes devant Dieu, une forme du thème de la commune humanité de tous les membres du corps social dont a vu l'importance. Il rappelle aussi l'ambivalence de la demande d'égalité qui se manifeste dans l'appel au respect: ces droits sociaux peuvent en effet tout aussi avoir pour sujet l'individu au principe de la théorie démocratique que prendre place dans une représentation holiste du social. Trois droits symbolisent le statut de citoyen pensé comme marque de la pleine appartenance sociale : le droit à la propriété d'une maison, le droit au travail et le droit de vote, matérialisé par la possession d'une carte d'électeur. Ils traduisent tous l'ancrage dans l'humanité définie par opposition à ce qui rappelle la condition d'esclave. L'ensemble des significations attachées au droit à la propriété d'une maison montre ainsi combien il est parfois vain de vouloir distinguer droits civils, droits politiques et droits sociaux. En ce qu'il représente à la fois la possibilité d'échapper au stigmate qui afflige celui qui n'a pas d'attaches fixes, la possibilité de disposer d'une adresse qui permet l'inscription sur les listes électorales et la possibilité d'accéder à des services urbains, ce droit participe de ces trois types de droit. Cela explique sans doute qu'il soit revendiqué avec autant d'ardeur que le droit au travail. Car, aujourd'hui encore, celui qui ne peut justifier d'une adresse permanente et d'un titre de travail lors d'un contrôle de police s'expose à être considéré comme un "vagabond" ou un "marginal» et court le risque d'un traitement discriminatoire. Mais, surtout, être propriétaire, c'est échapper au paiement d'un loyer qui grève le budget familial et à la vulnérabilité du locataire, toujours menacé de se retrouver à la rue au moindre revers de fortune. La mention du droit au travail comme droit du citoyen rappelle que, au Nordeste du Brésil, c'est longtemps le fait d'être rémunéré pour son labeur qui a distingué l'homme libre de l'esclave. Le choix d'une activité indépendante est ainsi fréquemment expliqué par le refus de relations de travail où la domination du patron sur l'employé s'apparente à l'assujettissement de l'esclave à son maître ${ }^{34}$. Le salariat déclaré n'en est pas moins fortement apprécié, en ce qu'il ouvre l'accès à la protection sociale, matérialisé notamment par la possession d'une carte de travail. Outre l'ouverture de droits sociaux, la carte de travail atteste de l'honorabilité sociale de son porteur. Preuve d'un emploi stable, elle permet d'acheter à crédit dans de nombreux commerces. Légalement reconnue comme document d'identité, elle est aussi fréquemment exigée lors de contrôles policiers où elle sert souvent à distinguer un "travailleur honnête » d'un "vagabond » ou d'un « marginal ». De plus, de la même façon que le paiement d'impôts locaux est considéré comme la contrepartie du droit à des services urbains de qualité, ceux qui acquittent des cotisations sociales estiment qu'elles doivent garantir des droits sociaux et, notamment, une retraite assurant une vie digne. S'il existe ici encore une obligation réciproque du citoyen et de l'État, on constate qu'elle ne renvoie pas à la 
possibilité du citoyen de participer aux affaires publiques. Bien que le droit de vote ne soit presque jamais cité parmi les droits du citoyen, il ne laisse nullement indifférent tant il exprime une forme symbolique d'intégration sociale en position d'égaux. Pour les analphabètes qui en ont été privés jusqu'en 1985, son obtention a mis fin à une situation d'infériorité juridique qui constituait aussi parfois un stigmate d'inaptitude au travail et a représenté bien plus la reconnaissance de leur pleine appartenance sociale que le pouvoir d'influer sur le gouvernement de la cité3 ${ }^{35}$. Les devoirs du citoyen Mais s'il a des droits, le citoyen a surtout des devoirs, tant et si bien que ceux qui « ne respectent pas les devoirs " (não cumprem os deveres) ne sauraient être considérés comme des citoyens. Il n'est de conversation prolongée sur ce que signifie « être un citoyen » sans que ne revienne le vocabulaire des obligations morales devant gouverner les relations entre parents, entre voisins, entre amis ou entre employeurs et employés. Est un citoyen celui qui est "droit », qui se montre respectueux de l'ordre social et concourt à son harmonie par son honnêteté et son ardeur au travail. Que des attributs moraux entrent dans la définition des devoirs attachés au statut de citoyen n'a en soi rien de surprenant. Un des aspects de la citoyenneté moderne renvoie à l'ensemble des qualités morales qui caractérisent le «bon citoyen» dans une société donnée ${ }^{36}$. L'absence de toute référence à la participation politique - l'aspect civique de la citoyenneté - est par contre notable. Sans doute faut-il y voir une conséquence directe de la faiblesse historique de l'exercice des droits politiques au Brésil. Le citoyen tel qu'on le conçoit à Brasília Teimosa est en fait essentiellement orientée vers la civilité, élément au cœur de la construction du concept de citoyenneté dans les démocraties modernes ${ }^{37}$. La civilité qui implique le sentiment de l'attachement à l'ordre social et à sa préservation en dépit des différences sociales, et concerne avant tout la vie en bonne intelligence des membres d'une même société, à la différence du civisme davantage orientée vers une participation active à la sphère publique. Aussi, sur la péninsule, le citoyen et le «bon citoyen » ne font qu'un. Les distinguer ne fait pas sens, puisque n'est citoyen que celui qui «remplit ses devoirs» vis-à-vis des différents collectifs auxquels il appartient. Qu'un individu vienne en revanche à manquer aux devoirs incombant au citoyen suffit à ce qu'il ne soit plus considéré comme tel. Tous ceux qui, par leurs actes, mettent en péril l'idéal d'une société harmonieuse et conviviale se voient refuser la qualité d'être humain (gente) associée à l'idée de citoyen, l'appartenance à la société signifiant l'appartenance à l'humanité. On considère ainsi parfaitement juste que des traitements dégradants qui révèlent la persistance des catégories du châtiment esclavagiste soient appliqués aux délinquants. Et y compris chez les sympathisants de formations engagées dans la défense des droits de l'homme, la population locale approuve sans réserve dans une écrasante majorité les passages à tabac, que l'on juge nécessaires à la manifestation de la vérité et à la punition des coupables ${ }^{38}$. Le cidadão est aussi un "bon Brésilien ", une identification à la nation qui rappelle que la citoyenneté exprime aussi, dans les sociétés modernes, un sentiment d'appartenance à une identité sociale globale qui diffère selon la spécificité de la dynamique historique de chaque pays ${ }^{39}$. Le lien social n'y réside plus dans des groupes primaires qui structurent le social, ni ne résulte d'un principe religieux ou dynastique qui unit les hommes; «le lien social [est] désormais essentiellement politique, c'est-à-dire national ${ }^{40}$. Et, dans la nation, le citoyen est défini comme un individu abstrait, dégagé de toutes appartenances et enracinements particuliers, qui se reconnaît dans des éléments identitaires perçus comme exclusivement liés à l'entité nationale. En témoigne, à Brasília Teimosa, l'engouement pour la Coupe du monde football où l'identification à l'équipe nationale est une autre 
façon d'affirmer le rôle essentiel du " peuple » dans l'affirmation de la nation ${ }^{41}$. Le fait, enfin, que le mot « citoyen " soit défini en termes moraux et ne soit que très rarement associé à l'idée de participation active aux affaires publiques ne signifie nullement que les citoyens ne puissent rien espérer des gouvernants. Bien au contraire, c'est justement parce que, en tant que Brésiliens, ils respectent strictement les normes nécessaires au bon fonctionnement de la société et à la préservation de l'ordre social qu'ils s'estiment fondés à attendre en retour des gouvernants des solutions à leurs difficultés. Au respect qu'ils manifestent doit correspondre le respect des politiciens, car si, eux, «remplissent leurs obligations ", à eux de respecter les leurs qui sont de reconnaître et de soulager la détresse de "ceux qui souffrent». C'est pourquoi beaucoup interprètent l'insuffisance de l'action et le manque d'égards des fonctionnaires envers les pauvres comme autant d'atteintes à leur qualité de citoyen. Les habitants de Brasília Teimosa ont du reste une idée très précise du rôle qui incombe au personnel politique : "trouver des solutions », "aider ", "régler les problèmes ». Autant de tâches qui, conformément à la conception du code de comportement que chacun doit observer selon sa place dans la société, constituent les "obligations », les « devoirs" des politiciens envers la population. Un «bon politicien » est un politicien qui «remplit ses devoirs", qui «travaille pour les pauvres», manifeste sa préoccupation pour leur sort en, selon une expression communément utilisée, les « regardant ». Une importance attachée au regard des élus qui révèle un autre aspect de la vulnérabilité de l'identité des pauvres urbains, incapables d'être assurés de leur pleine appartenance sociale sans la reconnaissance du supérieur. Que nous apprennent au total la centralité de l'exigence de respect dans ces représentations du statut de citoyen ? L'absence de toute mention à la participation politique nous met en présence d'une conception de la citoyenneté bien différente de celle de la théorie démocratique qui repose sur l'engagement des citoyens dans les affaires publiques. Les «droits » revendiqués ne participent de même guère de l'idée arendtienne du «droit à avoir des droits ": assurant à leurs bénéficiaires une existence digne, ils renvoient bien davantage au souvenir de formes anciennes de lien social exprimées dans un vocabulaire juridique. Car le citoyen est avant tout celui voit sa pleine appartenance sociale reconnue, celui qui se considère et est considéré comme un humain et ne peut être rapproché de ce qui évoque la condition de l'esclave tenu à la lisière de la société. La référence constante aux obligations morales qui garantissent le respect révèle une conception de la citoyenneté comme forme d'appartenance à une totalité ordonnée et harmonieuse où chacun, parce qu'il a sa place reconnue même en position subalterne, peut se prévaloir de prérogatives auprès des gouvernants.

2 L'enchevêtrement du lien social et du lien politique à Brasília Teimosa constitue une illustration de la difficile instauration de la démocratie au Brésil. L'ambivalence de sa population devant l'indétermination de l'époque en est l'élément premier. Manifeste dans les différents usages de la notion de respect, l'hésitation entre les formes anciennes de rapports hiérarchiques, qui garantissent aux pauvres une place dans l'ordre social, et une société d'égalité, qui suppose la similitude entre les hommes et donne à chacun la possibilité d'accéder aux positions socialement valorisées, se répercute sur le déchiffrement des activités politiques. Cette exigence de respect entrave l'institution politique du social, ce que Claude Lefort appelle le rôle instituant du politique dans la "mise en sens » et la "mise en scène » des rapports sociaux. Le politique étant perçu au travers d'obligations morales énonçant les fondements du lien social, l'activité politique n'est jamais perçue comme un domaine autonome de la vie 
sociale. Ou, comme lors de ce moment fondateur qui vit la reconnaissance de la détresse du peuple dans les années 1930-1950, elle n'est que pur rapport social, l'action des gouvernants étant interprétée comme procédant du devoir des couches supérieures envers les groupes subalternes et, dans ce cas, lien social et lien politique ne font qu'un. $\mathrm{Ou}$, comme aujourd'hui où l'incapacité des élus à perpétuer cette forme de légitimation inaugurée sous le régime autoritaire de Getúlio Vargas provoque le discrédit du personnel et des institutions politiques, elle est vue comme totalement séparée de la vie respectable à laquelle aspirent les secteurs populaires, qui doivent s'en garder sous peine d'y perdre une dignité fondée sur et conquise par le travail. En l'absence d'une amélioration notable de la condition des plus démunis attribuable à l'action gouvernementale, rien ne paraît pouvoir restaurer la confiance en la sphère politique. L'effondrement de la capacité d'intégration de l'État dans un pays où il avait joué un rôle moteur dans l'institution de la nation produit en outre un sentiment d'étiolement du lien social : non seulement les inégalités sociales n'ont pas diminué depuis la fin de l'autoritarisme, mais, surtout, la quasi disparition des importantes possibilités de mobilité naguère offertes et la croissance de phénomènes de désorganisation ébranlent en profondeur des mythes fondateurs de la nation, qui permettaient à tous les Brésiliens, quelles que soient l'ampleur des disparités sociales, de se penser comme membres d'une totalité harmonieuse en progrès continu et promise à la prospérité. Le cas de Brasília Teimosa est à ce sujet exemplaire en ce qu'il révèle l'incapacité des acteurs gouvernementaux à proposer des perspectives d'amélioration des conditions de vie une fois que, l'urbanisation du site réalisée, les difficultés de ses habitants résident essentiellement dans la faiblesse du pouvoir d'achat, un taux élevé de chômage, l'insuffisance des systèmes scolaires et de santé publique, problèmes autrement plus complexes et difficiles à résoudre, a fortiori dans un contexte de crise fiscale et de croissance limitée. L'adhésion de sa population à l'imaginaire de la démocratie moderne est ainsi d'autant plus difficile que la sortie de l'autoritarisme s'est produite dans un contexte de crise économique qui voyait se dégrader ses conditions de vie ${ }^{42}$. Et les élections, au cœur de la symbolique démocratique, constituent moins un moment de l'exercice de la citoyenneté qu'un temps particulier («l'époque de la politique ») lors duquel les candidats reconnaissent la pleine appartenance sociale des pauvres en venant solliciter leurs suffrages. Si l'on accepte avec Jean Leca de voir les trois traits essentiels de la citoyenneté dans la croyance en l'intelligibilité du monde politique, l'empathie envers les autres citoyens et les gouvernants, qui suppose la capacité à prendre en considération leurs préférences et leurs contraintes, et la civilité, qui permet de gérer la tension entre l'appartenance commune et la différenciation sociale, la difficulté de l'exercice de la citoyenneté par les habitants de Brasília Teimosa n'en ressort que davantage ${ }^{43}$. Non seulement le discrédit des activités politiques ne les incite guère à s'y intéresser, mais, en plus, les changements fréquents de partis des politiciens, la fluidité des alliances politiques et les caractéristiques du système électoral achèvent de brouiller l'intelligibilité de la sphère politique. Quand l'action publique n'est perçue que comme un problème de «bonne volonté » et de «bon cœur » des gouvernants, la propension à mettre en œuvre un raisonnement empathique à leur égard, et en particulier de comprendre les limites imposées par la diminution des recettes fiscales en temps de crise économique et d'endettement public, est singulièrement limitée. Seul l'attachement à la civilité - manifestée dans le besoin d'affiliation aux couches favorisées et dans l'exigence du châtiment impitoyable des délinquants qui, par leurs actes, se sont placés hors des frontières de la société - se 
retrouve dans la définition du statut de citoyen. Au point de la saturer. On aurait néanmoins tort de ne voir dans ces représentations du statut de citoyen aucune parenté avec l'idée moderne de citoyenneté. En témoignent notamment les demandes adressées à la puissance publique au nom de l'appartenance à la nation. Car si le statut de citoyen ne passe pas par la participation politique, il a bien le territoire national pour cadre premier et l'État pour garant. Mais dans un pays où le lien politique entre le pouvoir et le peuple se tisse sous un régime autoritaire par l'adoption d'une législation professionnelle corporatiste, la dimension politique de la citoyenneté n'apparait pas. Néanmoins, si les analyses qui précèdent ne laissent guère entrevoir la pleine adhésion des secteurs populaires à l'imaginaire de la démocratie moderne, on peut néanmoins espérer que les transformations entraînées par l'urbanisation et par l'élévation considérable du niveau d'instruction susciteront tôt ou tard une affirmation plus revendicative et autonome de la citoyenneté chez les plus démunis des Brésiliens, surtout représentés jusque là par des membres de groupes favorisés peu disposés à penser un projet d'amélioration de la condition des couches subalternes qui se traduirait par une dégradation de leur propre position sociale.

\section{NOTES}

1. Cet article repose les résultats de deux recherches distinctes à Brasília Teimosa. La première, menée de 1992 à 1994, a porté sur les rapports sociaux, le rôle des associations d'habitants et les pratiques politiques. Cf. Dominique Vidal, La politique au quartier. Rapports sociaux et citoyenneté à Recife, Paris, Éditions de la Maison des sciences de l'homme, 1998. Fruit d'une autre enquête de terrain aux mois de juillet et août 1998, la seconde s'est intéressée aux modes d'entrée dans l'âge adulte et aux formes d'individualisation chez les jeunes de la péninsule. Toutes deux reposent sur une approche ethnographique du terrain, fondée notamment sur cinq mois de résidence in situ et un cours d'initiation à la langue française donné au siège du Conseil des habitants. C'est en effet seulement après un contact prolongé avec le milieu étudié que je découvris l'importance du vocabulaire moral de la quotidienneté dans les propos sur les activités politiques et, en particulier, la centralité de la notion de respect, indice de l'enchevêtrement du lien social et du lien politique.

2. Alors qu'elle est l'objet d'une réflexion spécifique en philosophie morale (cf. par exemple l'ouvrage collectif dirigé par Catherine Audard, Le respect. De l'estime à la déférence : une question de limite, Paris, Éditions Autrement, 1993), la notion de respect en tant que telle n'a guère suscité l'intérêt des chercheurs en sciences sociales qui l'ont souvent rapprochée de la notion d'honneur telle qu'elle a été étudiée dans les sociétés méditerranéennes. Dans cette perspective, on peut notamment citer en ce qui concerne le Brésil les travaux de Claudia Fonseca, « La violence et l'honneur : le code d'honneur dans un bidonville brésilien », Les Temps modernes, 455, juin 1984, pp. 2193-2235 et de Marcos Alvito Pereira de Souza, «A honra do Acari », in Gilberto Velho \& Marcos Alvito Pereira de Souza (éd.), Cidadania e violência, Rio de Janeiro, Editora UFRJ/Editora FGV, 1996, pp. 147-164. Bien que nous partageons nombre de leurs 
analyses, nous estimons toutefois qu'on ne saurait confondre ces deux notions dans des sociétés modernes et individualisées où, à la différence du monde méditerranéen, l'identité n'est plus directement donnée par la structure sociale. Nous espérons d'ailleurs à ce sujet publier prochainement avec Patrícia Sampaio Silva les résultats d'une recherche socio-historique en cours sur la différence entre l'honneur et le respect dans l'État de Pernambuco au Nordeste du Brésil.

3. Cf. Philippe Bourgois, In Search of Respect. Selling Crack in El Barrio, Cambridge, Cambridge University Press, 1995.

4. Il ne sera toutefois pas question dans ce texte des usages de la notion de respect chez les jeunes délinquants ou les prostituées.

5. Nous nous inspirons en cela de la démarche préconisée par Clifford Geertz qui fait de la capacité du chercheur à « voir les choses du point de vue de l'indigène » une condition essentielle de la qualité de l'interprétation, « Du point de vue de l'indigène : sur la nature de la compréhension anthropologique », Savoir local, Savoir global, Paris, PUF, 1986 [1983], pp. 71-90.

6. L'histoire de ces organisations révèle cependant que la mobilisation de sa population ne s'est produite que pendant une période relativement limitée (de 1979 à 1984) pendant la transition démocratique et n'a surtout concerné qu'une minorité active de dirigeants associatifs. Cf. Dominique Vidal, op. cit., pp. 85-130.

7. Les Italo-Américains, les juifs des ghettos et les ouvriers anglais des premières décennies de ce siècle en sont probablement les figures les plus connues. Cf. William Foote Whyte, Street Corner Society. La structure sociale d'un quartier italo-américain, Paris, La découverte, 1995 [1943] ; Herbert J. Gans, The Urban Villagers. Group and Class in the Life of Italo-Americans, New York, The Free Press, 1962 ; Richard Hoggart, La culture du pauvre. Étude sur le style de vie des classes populaires en Angleterre, Paris, Éditions de Minuit, 1970 [1957] ; Louis Wirth, Le ghetto, Grenoble, Éditions du champ urbain, 1980 [1928].

8. La plage n'est pas à Recife le théâtre de scènes de violence juvénile qui exprime les difficultés d'intégration à la société de masse des jeunes de milieux populaires. Cf. Angelina Peralva, «Démocratie et violence : le cas de Rio de Janeiro », Problèmes d'Amérique latine, n 23, octobre-décembre 1996, pp. 79-98.

9. Cf. Herbert J. Gans, op. cit., p. 221.

10. Katia M. de Queirós Mattoso, Être esclave au Brésil : 16ème - 19ème siècle, Paris, L'Harmattan, 1994 [1979], p. 117.

11. Comme le souligne Dominique Schnapper dans un essai sur la nation démocratique, l'affirmation de l'appartenance nationale procure aux individus en situation d'infériorité une forme d'égalité symbolique avec les autres citoyens qui n'existe pas dans la vie sociale. Cf. Dominique Schnapper, La communauté des citoyens. Sur l'idée moderne de nation, Paris, Gallimard, 1993.

12. Cf. à ce sujet l'ouvrage de Daniel Pécaut, Entre le Peuple et la Nation. Les intellectuels et la politique au Brésil, Paris, Éditions de la Maison des sciences de l'homme, 1989.

13. Maria Isaura Pereira de Queiroz, Carnaval brésilien : le vécu et le mythe, Paris, Gallimard, 1992.

14. Ibid., p. 140.

15. Ferdinand Tönnies, Communauté et société. Catégories fondamentales de la sociologie pure, Paris, Retz-CEPL, 1977 [1887], p. 56. 
16. Il s'agit là incontestablement de l'apport spécifique de l'approche ethnographique sur les autres formes d'enquête en sciences sociales. À la différence de recherches uniquement fondées sur la passation de questionnaires et des entretiens standardisés, l'observation de la population étudiée sur une longue durée et les propos recueillis dans des situations informelles une fois des liens de connaissance établis ont en effet fait apparaître des pratiques et des faits occultés lors des premières rencontres.

17. Claudia Fonseca, «Alliés et ennemis en famille », Les Temps modernes, 499, février 1988, pp. 28-58.

18. Larissa Adler Lomnitz, Networks and Marginality. Life in a Mexican Shantytown, New York, Academic Press, 1977 [1976].

19. Oscar Lewis, La Vida. Une famille portoricaine dans la culture de la pauvreté : San Juan et New York, Paris, Gallimard, 1969 [1965] ; Les enfants de Sanchez.

Autobiographie d'une famille mexicaine, Paris, Gallimard, 1981 [1961].

20. À ce propos, il conviendrait de se demander dans une recherche sur les couches moyennes et supérieures brésiliennes si, en définitive, la perception des groupes sociaux en termes de « eux » et " nous » n'est pas nettement plus marquée chez les membres de ces secteurs, qui assignent communément les «pauvres » et le «peuple » dans une irréductible altérité, que parmi les populations démunies qui puisent de nombreux éléments d'identification dans les milieux socialement favorisés.

21. La propension à refuser de se considérer Noir est plus forte chez les femmes que chez les est plus forte chez les femmes que chez les hommes. Celles-ci estiment souvent appartenir à la catégorie des mulatas, métisses idéalisées dans l'imaginaire sexuel des Brésiliens.

22. Cf. Michel Agier, «Ethnopolitique. Racisme, statut et mouvement noir à Bahia », Cahiers d'études africaines (Paris), XXXII (1), n 125, 1992, pp. 53-81.

23. L'inachèvement des procédures de légalisation de l'usage du sol, les problèmes créés par les dysfonctionnements du réseau d'égouts, de la collecte des ordures et du système d'adduction d'eau, et la présence de plusieurs dizaines de baraques sur pilotis sur la plage sont, au demeurant, autant d'éléments susceptibles de maintenir cet espace dans la catégorie « favela » au regard des normes de l'Institut brésilien de géographie et de statistiques (IBGE). Brasília Teimosa n'a du reste n'a jamais cessé d'être considérée comme une favela par la population de Recife, toutes couches sociales confondues, qui tient pour négligeables les transformations intervenues et rappelle l'étroitesse des rues, l'entassement des maisons et la pauvreté régnante. Le fait d'avoir longtemps abrité des maisons de tolérance vaut d'ailleurs une réputation déplorable à ses résidents dont beaucoup ont déjà lu, une fois révélé leur lieu d'habitation, la méfiance sur le visage de leurs interlocuteurs.

24. Tenue comme un haut lieu de la participation populaire dans la littérature sur les mouvements sociaux urbains à Recife, Brasília Teimosa doit en réalité son urbanisation à l'action d'une minorité active de militants associatifs, conseillée par des professionnels du droit et de l'urbanisme issus des classes moyennes dans le contexte de transition à la démocratie où, dans un souci de légitimation, les responsables politiques du parti soutenant le régime militaire - l'ARENA - entendent mettre en œuvre une politique urbaine plus favorable aux secteurs populaires. Un examen de quelques sources écrites disponibles (archives du Conseil des habitants, documents administratifs, presse) révèle ainsi que jamais plus de 300 habitants ont participé aux quelques manifestations pour l'urbanisation de la péninsule et que le projet qui en prévoit les modalités a été financé par le gouvernement de l'État de Pernambuco, alors 
dirigé par Marco Maciel, un des chefs de file de l'ARENA. Si toute une étude reste à faire sur l'écart entre la réalité des mouvements sociaux dans ces dernières années de l'autoritarisme et les interprétations souvent très idéologiques qui en ont été données, il n'en reste pas moins que les discours tenus sur la mobilisation de Brasília Teimosa ont eu pour conséquence bien réelle d'être appropriés par ses habitants pour se penser comme plus « avancés » que les résidents d'autres espaces déshérités.

25. Richard Hoggart, op. cit., pp. 347-376.

26. Paul Willis, «L'écoles des ouvriers ", Actes de la recherche en sciences sociales, 24, novembre 1978, pp. 50-61 ; Learning to Labor. How Working Class Kids Get Working Class Jobs, New York, Columbia University Press, 1981 [1977].

27. Sur le paternalisme au Brésil, voir le numéro spécial de la revue Lusotopies, Éditions Karthala, 1996.

28. Pour un commentaire approfondie de cette idée chez Tocqueville, on lira l'admirable texte de Marcel Gauchet, « Tocqueville, l'Amérique et nous. Sur la genèse des sociétés démocratiques ", Libre, 7, 1980, pp. 43-120.

29. Dans des travaux connus et souvent cités, l'anthropologue Roberto DaMatta a également montré comment, au Brésil, un code hiérarchique et un code égalitaire opéraient simultanément dans nombre de pratiques. Cf. Roberto DaMatta, Carnavals, bandits et héros. Ambiguïtés de la société brésilienne, Paris, Éditions du Seuil, 1983 [1978] ; A casa e a rua. Espaço, cidadania, mulher e morte no Brasil, Rio de Janeiro, Editora Guanabara, 1991 [1985]; Conta de mentiroso : sete ensaios de antropologia brasileira, Rio de Janeiro, Rocco, 1993. Notre analyse des liens complexes de la notion de respect avec les idées de hiérarchie et d'égalité diffère néanmoins considérablement de la sienne. Si DaMatta voit dans la coexistence d'un code hiérarchique et d'un code égalitaire un principe central d'explication de la société brésilienne, notre interprétation insiste au contraire sur son indétermination au moment où la notion de respect a été appréhendée, à savoir lors de la proclamation des idéaux égalitaires et individualistes de la démocratie moderne dans un contexte de désorganisation et de déstructuration des formes anciennes de lien social. À l'approche résolument ahistorique de DaMatta, nous préférons, par la prise en compte des effets de ces changements sur les représentations sociales et politiques de la population étudiée, souligner la fragilité des configurations où se rencontrent le principe hiérarchique et l'idée d'égalité. C'est notamment pourquoi différentes formes d'égalité sont distinguées.

30. Bien que nous ne disposions d'aucune étude lexicographique sur l'histoire et les usages du mot cidadão au Brésil, tout indique que ce mot est utilisé dans des sens différents selon les époques, les régions, les milieux et les contextes sociaux. Aussi ne peut-on que s'étonner des propos de Roberto DaMatta qui soutient que « le mot "citoyen" est toujours utilisé dans des situations négatives au Brésil, pour marquer la position de quelqu'un qui se trouve en désavantage ou même en infériorité ", cf. Roberto DaMatta, A casa e a rua. Espaço, cidadania, mulher e morte no Brasil, op.cit., p. 86. Sans doute fonde-t-il son analyse uniquement sur l'observation des membres des couches moyennes et supérieures pour lesquels le mot cidadão possède souvent une connotation péjorative et exprime un doute quant à l'identité et l'honnêteté de celui qu'il vient désigner. Le sens de ce mot voisine alors avec celui parfois attaché au mot « individu » en français quand on parle de « cet individu » pour marquer une distance vis-à-vis de quelqu'un, connu ou non, mais suspect.]. Les droits du citoyen Plus que de Droit, il est souvent question des « droits » à Brasília Teimosa. La distinction n'est pas 
formelle ; elle informe même en profondeur sur le sens donné au statut de citoyen. Car, comme chez les habitants d'un quartier populaire de São Paulo étudiés par Teresa Caldeira, le mot « droit » revêt une signification différente selon qu'il est employé au singulier ou au pluriel[[Teresa Caldeira, A política dos outros. O cotidiano dos moradores da periferia e o que pensam do poder e dos poderosos, São Paulo, Editora Brasiliense, 1984.

31. Arrivé au pouvoir par un coup d'État en 1930, dictateur sous l'Estado Novo (1937-1945) au cours duquel se met en place cette législation professionnelle, Vargas doit quitter la présidence de la République en 1945. Démocratiquement élu cette fois, il la retrouve en 1950 avant de se suicider en 1954 suite à une très dure campagne de l'opposition. Un geste que beaucoup interpréteront comme un sacrifice pour le peuple. Idée de sacrifice au nom de l'intérêt du peuple que, du reste, Vargas exprime lui-même dans la « Lettre-testament » qu'il laisse avant de mettre fin à ses jours.

32. Pour ceux qui ont vécu, même enfants, dans les campagnes de Pernambuco et, surtout, dans la zone de culture de la canne à sucre au tournant des années 1960, les «droits » renvoient aussi à la législation professionnelle qui réglemente les rapports entre travailleurs agricoles et propriétaires terriens. À l'instar du droit du travail qui se met en place sous Getúlio Vargas, ces « droits » apparaissent résulter d'un « don » du gouverneur de l'époque, le dirigeant populiste Miguel Arraes. Cf. sur le sujet les travaux réalisés par les anthropologues du Museu Nacional de la Universidade Federal de Rio de Janeiro : Lygia Sigaud, Os clandestinos e os direitos. Estudo sobre trabalhadores da cana de açúcar de Pernambuco, São Paulo, Duas Cidades, 1979 et « Des plantations aux villes : les ambiguïtés d'un choix ", Études rurales, pp. 131-132, juillet-décembre 1993 : 19-37 ; Rosilene Alvim et José Sérgio Leite Lopes, «Familles ouvrières, familles d'ouvrières ", Actes de la recherche en sciences sociales, n 84, pp. 78-84, 1990 ; Afrânio Garcia, Libres et assujettis. Marché du travail et mode de domination au Nordeste, Paris, Éditions de la Maison des sciences de l'homme, 1989.

33. Dans un article souvent cité dans la littérature sur les mouvements sociaux urbains, Eunice Durham affirmait ainsi : «nous assistons à un processus de construction d'un ensemble de droits par les mouvements sociaux. Et cela ne se produit pas au travers d'une codification complète et achevée d'une réalité existante, mais comme le revers d'une définition cumulative de carences qui sont définies comme inacceptables ». Cf. Eunice Durham, « Movimentos sociais : a construção da cidadania », Novos Estudos Cebrap, $\mathrm{n}^{\circ}$ 1, 1984, p. 19.

34. La même crainte du rapport de dépendance inhérent au travail salarié a été relevé aux États-Unis. Cf. Judith Shklar, La citoyenneté américaine. La quête de l'intégration, Paris, Calmann-Lévy, 1991.

35. C'est pourquoi il importe de ne pas interpréter les importants taux d'abstention, de vote nul et de vote blanc en milieu populaire comme un désintérêt pour le droit de vote, ces comportements reflétant avant tout le doute généralisé sur la participation politique. L'on sait que, aux États-Unis et en France, le contraste entre la vigueur des luttes pour l'accès au suffrage et la faiblesse de la pratique électorale manifeste l'importance de l'acquisition du droit de vote comme reconnaissance de la pleine appartenance à la société et de la qualité de citoyen comme statut social. Cf. Judith Shklar, op. cit. et Pierre Rosanvallon, Le sacre du citoyen. Histoire du suffrage universel en France, Paris, Gallimard, 1992.

36. Jean Leca, « Questions on citizenship », in Chantal Mouffe (dir.), Dimensions of Radical Democracy. Pluralism, Citizenship, Community, London, Verso, 1992, p. 18. 
37. Jean Leca, « Individualisme et citoyenneté », in Pierre Birnbaum \& Jean Leca (dir.), Sur l'individualisme, Paris, Presses de la FNSP, pp. 159-209 ; Edward C. Banfield, Civility and Citizenship in Liberal Democratic Societies, New York, Paragon House, 1992.

38. L'écart entre le discours et la pratique étant néanmoins, là encore, particulièrement accentué, ces propos n'empêchent pas pour autant des délinquants notoires de jouir d'une large estime pour leurs qualités de « bons voisins ", pourvu qu'ils se montrent charitables, polis et se livrent à leurs activités hors de la péninsule.

39. Guy Hermet, «Des concepts de la citoyenneté dans la tradition occidentale ", in Daniel Pécaut et Bernardo Sorj (dir.), Métamorphoses de la représentation politique au Brésil et en Europe, Paris, Éditions du CNRS, 1991, pp. 19-29.

40. Dominique Schnapper, op. cit., p. 15.

41. Sur le football, les secteurs populaires et la construction de l'identité nationale, voir José Sérgio Leite Lopes et Sylvain Maresca, « La disparition de 'la joie du peuple'. Notes sur la mort d'un joueur de football », Actes de la recherche en sciences sociales, $\mathrm{n}^{\circ} 79$, septembre 1989, pp. 21-36, et José Sérgio Leite Lopes et Jean-Pierre Faguer, «L'invention du style brésilien. Sport, journalisme et politique au Brésil », Actes de la recherche en sciences sociales, $n^{\circ} 103$, juin 1994, pp. 27-35.

42. L'émergence d'une conscience démocratique chez les populations pauvres sur laquelle insistaient de nombreux auteurs qui raisonnaient en termes de mouvements sociaux pendant la transition démocratique paraît aujourd'hui un thème daté d'un autre temps. Toutes les études révèlent d'ailleurs aujourd'hui le peu d'intérêt pour la politique, l'ampleur du discrédit des élus, et de la désaffection des institutions politiques en milieu populaire.

43. Jean Leca, art. cité.

INDEX

Index géographique : Amérique du Sud, Brésil

Mots-clés : pauvreté, politique, exclusion 